\title{
26464 - COMPARISON OF THE BONFILS FIBRESCOPE AND BULLARD LARYNGOSCOPE
}

\section{Quoc Nguyen MD, Kevin Armstrong, MD; University Of Western Ontario, London, ONTARIO, Canada}

\section{INTRODUCTION:}

Difficult or awkward laryngoscopy and intubation occurs in approximately $3 \%$ of anesthetic cases but remains an important cause of anesthesia morbidity and mortality $(1,2)$. Despite the abundance of available airway devices, the majority of anesthesiologists have shown reluctance to adopt them (3). The Bonfils(Alt + 0153) intubation fiberscope and Bullard(Alt + 0153) laryngoscope are devices suitable for managing the difficult airway when used by experienced operators $(4,5)$. This study compared the use of the Bonfils and Bullard in novice users and their preference for either device. The Laerdal AirMan(Alt + 0153) simulator provided a reproducible setting to compare these devices.

\section{METHODS:}

After ethics review board approval, 40 anesthesia providers (subjects) were recruited and consented to participate in this comparison. Following randomization to determine order of use, 10 consecutive intubations, five per device, were performed on the Laerdal AirMan intubating mannequin. Limited cervical spine movement was simulated and ceramic upper dentition was present. Time to intubation (TTI) was the primary endpoint and defined as the elapsed time from introduction of the device into the mouth to successful endotracheal tube ventilation. A failure was recorded after the third unsuccessful attempt or after 90 seconds had elapsed. Secondary endpoints included failed and esophageal intubation, damage to dentition and instruments, and preference for a device.

\section{RESULTS:}

Median TTI with the Bonfils was significantly slower at $48.3 \mathrm{~s}$ (33.5 - 65.7) compared with the 33.7 (27.9 - 42.6) as seen with the Bullard. A learning effect was demonstrated with both devices. By the fifth attempt there was no difference in TTI. An increased rate of failed intubation, esophageal intubation, airway trauma, and equipment damage was seen with the Bonfils.

\section{CONCLUSION:}

Our results suggest that both the Bullard and Bonfils are effective in this stimulated difficult airway setting. The Bullard scope resulted in shorter TTI with lower rates of failed intubation, esophageal intubation, airway trauma, and equipment damage. A learning effect was observed with successive attempts with both devices. Improvements in technique acquired through repetition were demonstrated by the improvements in TTI. Although improvements were seen with successive attempts, more users preferred the Bullard at the end of the trial due in part to their prior experience with it. The simulator is a potentially valuable tool to overcome the barriers incumbent to the introduction of any airway device by providing a realistic setting for anesthesiologists to learn and practice new techniques without compromising patient care. An assumption is made that these results are applicable to the clinical situation.

\section{REFERENCE:}


1. Can J Anes 2004: 51:R6

2. Anes 1990: $72 ; 828$

3. Can J Anes 2002; 49: 850-6

4. J Clin Anes 1991; 3: 473-7

5. Anaes 2004; 59:1207-09

Table 1: Median TTI with interquartile range for all attempts and attempt number 5, division of TTI at preset intervals, conplication rates, and user evaluation of the airway devices.

\begin{tabular}{|c|c|c|c|}
\hline & Bonfils & Bullard & p value \\
\hline IIf (sec.) & $48.3(33.5-65.7)$ & $33.7(27.9-42.6)$ & 0.011 \\
\hline TTI attempt is $(\mathrm{sec})$. & $33.0(17.0-48.2)$ & $22.7(20.0-29.4)$ & 0.242 \\
\hline $\mathrm{TTI}<30$ sec. & 72200 & 99200 & 40001 \\
\hline TTI $<60$ sec. & $135 / 200$ & $169 / 200$ & $\infty 001$ \\
\hline TII $<90$ sec. & 159200 & 186200 & 20001 \\
\hline$T \pi \geq 90$ sec. (failure) & $41 / 200$ & $14 / 200$ & 4001 \\
\hline Esoplageal infubatiog & 12 & 2 & $\infty 0001$ \\
\hline Devalal injuy & 4 & 0 & 0.116 \\
\hline Equipment damage & 8 & 0 & 0.005 \\
\hline User rating Easy & 22 & 33 & 8001 \\
\hline User rating: Diffscult & 18 & 7 & $\infty 01$ \\
\hline Preferred Desice & 7 & 23 & 001 \\
\hline
\end{tabular}

